\title{
Analysis of plasma free amino acid profiles in canine brain tumors
}

\author{
SHINICHI UTSUGI ${ }^{1,2^{*}}$, KAZUO AZUMA $^{3 *}$, TOMOHIRO OSAKI $^{3}$, YUSUKE MURAHATA $^{3}$, \\ TAKESHI TSUKA ${ }^{3}$, NORIHIKO ITO ${ }^{3}$, TOMOHIRO IMAGAWA ${ }^{3}$ and YOSHIHARU OKAMOTO ${ }^{3}$ \\ ${ }^{1}$ Department of Clinical Veterinary Medicine, The United Graduate School of Veterinary Science, Yamaguchi University, \\ Yamaguchi 753-8515; ${ }^{2}$ Department of Neurology, Japan Animal Referral Medical Center, \\ Kawasaki, Kanagawa 213-0032; ${ }^{3}$ Department of Clinical Medicine, School of Veterinary Medicine, \\ Faculty of Agriculture, Tottori University, Tottori 680-8553, Japan
}

Received July 27, 2016; Accepted October 5, 2016

DOI: $10.3892 /$ br.2016.825

\begin{abstract}
Canine brain tumors are best diagnosed using magnetic resonance imaging (MRI). However, opportunities of MRI examination are restricted due to its limited availability in veterinary facilities; thus, numerous canine brain tumors are diagnosed at an advanced stage. Therefore, development of a noninvasive diagnostic biomarker is required for the early detection of brain tumors. In the present study, plasma free amino acid (PFAA) profiles between dogs with and without brain tumors were compared. A total of 12 dogs with brain tumors, diagnosed based on clinical signs, and on the results of intracranial MRI and/or pathological examination were evaluated. In addition, eight dogs diagnosed with idiopathic epilepsy and 16 healthy dogs were also included. A liquid chromatography system with automated pre-column derivatization functionality was used to measure the levels of 20 amino acids. As a result, the levels of three amino acids (alanine, proline and isoleucine) were increased significantly (1.6-, 1.5- and 1.6-fold, respectively) in the plasma of dogs with brain tumors as compared with the levels in control dogs (all $\mathrm{P}<0.05)$. Thus, the PFAA levels of dogs with brain tumors differed from those of healthy dogs. The present study demonstrated that analysis of PFAA levels of dogs with brain tumors may serve as a useful biomarker for the early detection of canine brain tumors.
\end{abstract}

\section{Introduction}

Brain tumors in animals are more commonly observed in dogs rather than in cats, and are frequently detected in middle-aged to geriatric dogs (1). The incidence of canine brain tumors has

Correspondence to: Professor Yoshiharu Okamoto, Department of Clinical Medicine, School of Veterinary Medicine, Faculty of Agriculture, Tottori University, 4-101 Koyama-Minami, Tottori 680-8553, Japan

E-mail: yokamoto@muses.tottori-u.ac.jp

*Contributed equally

Key words: brain tumor, dog, glioma, meningioma, plasma free amino acid been reported to be $4.5 \%$ globally (2). The most common type of canine brain tumors is meningioma, followed by glioma (including astrocytoma and oligodendroglioma) (2-4). As canine brain tumors are not detectable by routine blood analysis or X-ray examination of the skull, they are best diagnosed using magnetic resonance imaging (MRI) (5). However, opportunities of MRI examination for canines are limited due to the high cost of this technique and its limited availability in veterinary facilities. The majority of brain tumors are not suspected until after clinical signs appear, and it is difficult to acquire MRI scans immediately in dogs with suspected brain tumors. Furthermore, numerous canine brain tumors are diagnosed at an advanced stage. Thus, development of a noninvasive diagnostic biomarker with high sensitivity and specificity is required for the early detection of canine brain tumors.

In human medicine, recent studies have reported that the levels of plasma free amino acid (PFAA) profiles are altered in certain diseases, such as in cancer (including pancreatic, breast and lung cancers) and cardiovascular diseases, and that these changes can predict future development of disease $(6,7)$. Therefore, it has been considered that PFAA profiles may be used as efficient disease biomarkers. In veterinary medicine, it has also been reported that PFAA levels are altered in dogs with malignant mammary tumors, oral melanoma and lymphoma (8-10). In addition, it has been reported that the levels of glutamic acid in the CSF are increased in dogs with idiopathic epilepsy (11).

Various metabolites, such as amino acids and choline, have been analyzed in human glioma patients by means of proton magnetic resonance spectroscopy (MRS) or from tissue specimens obtained during surgery or autopsy $(12,13)$. However, there have been few reports on PFAA analysis in intracranial disorders (14). To the best of our knowledge, the association between PFAA levels, canine brain tumors and canine idiopathic epilepsy has not been investigated thus far. Therefore, the present study investigated the PFAA level profiles in dogs with brain tumors and compared the data with healthy control dogs and dogs with idiopathic epilepsy.

\section{Materials and methods}

Animals. A total of 12 dogs with brain tumors brought to the Animal Medical Center of Tottori University and Japan 
Animal Referral Medical Center from October 2014 to June 2015 were evaluated in the current study. Diagnosis of brain tumors was based on clinical signs, and intracranial MRI and/or pathological examination. Generalized seizure, changes of character, depression, circling and tetraparesis were common clinical signs. MRI was performed using a 0.4T unit (AIRIS; Hitachi Medico, Tokyo, Japan) and 1.5T unit (Echelon Vega; Hitachi Medico, Tokyo, Japan). In all dogs, T1-, T2- and contrast-enhanced T1-weighted images were obtained. The age at diagnosis ranged between 7 and 14 years, with a mean age of 10.5 years. Six dogs were male and six dogs were female. A total of 6 dogs were suspected with glioma, 4 dogs were suspected or confirmed to suffer from meningioma, and 2 dogs had metastatic brain tumor of the mammary gland. In addition, 8 dogs diagnosed with idiopathic epilepsy were included, with an age at diagnosis ranging between 3 and 10 years (mean age, 5.5 years). A total of 16 healthy control dogs were also included in the study, with an age ranging between 1 and 14 years (mean age, 8.1 years). All information concerning these dogs is summarized in Table I. The study protocol was approved by the Ethics Committee on Animal Trials of the Japan Animal Referral Medical Center (Tokyo, Japan).

Blood collection. The dogs were fasted for $8 \mathrm{~h}$ before blood collection. Venous blood was collected in tubes containing heparin and was immediately centrifuged at $1,700 \mathrm{x} \mathrm{g}$ for $15 \mathrm{~min}$ at $4^{\circ} \mathrm{C}$. Following the centrifugation, plasma was promptly removed and frozen at $-80^{\circ} \mathrm{C}$ until required for PFAA measurements. The plasma was deproteinized in methanol [plasma:methanol (v/v), 1:9] for $20 \mathrm{~min}$, the samples were then centrifuged at $15,000 \times \mathrm{g}$ for $10 \mathrm{~min}$ at $4^{\circ} \mathrm{C}$, and the supernatant fluid was obtained.

PFAA measurements. Analysis of PFAA levels in the plasma of the dogs was performed using a pre-column technique with liquid chromatography according to previously reported methods (15). All reagents, including standard amino acid solutions (type $\mathrm{H}$ ), were purchased from Wako Pure Chemical Industries Ltd. (Osaka, Japan). A liquid chromatography system with automated pre-column derivatization functionality was used in the analysis (Nexera X2; Shimadzu Corporation, Kyoto, Japan). A total of 20 compounds were measured in the analysis, including the following basic amino acids and associated molecules: Alanine (Ala), arginine (Arg), asparagine (Asn), aspartic acid (Asp), cysteine-cysteine (Cys-Cys), glutamic acid (Glu), glutamine (Gln), glycine (Gly), histidine (His), isoleucine (Ile), leucine (Leu), lysine (Lys), methionine (Met), phenylalanine (Phe), proline (Pro), serine (Ser), threonine (Thr), tryptophan (Trp), tyrosine (Tyr) and valine (Val). Plasma levels of the amino acids are expressed in $\mu \mathrm{mol} / \mathrm{l}$.

Statistical analysis. Data are expressed as the mean \pm standard deviation. All figures were prepared by Excel 2013 (Microsoft Japan Co., Ltd., Tokyo, Japan). Differences between groups were evaluated using the Steel-Dwass test (4-Step Excel Statistics version 3; OMS publishing Inc., Tokyo, Japan). $\mathrm{P}<0.05$ was considered to indicate a statistically significant difference. The data are presented in radar charts. The radar charts show the percentage of the mean PFAA levels in dogs with brain tumors and idiopathic epilepsy, as compared to the control groups (taken as 100\%).

Immunohistochemical analysis. Immunohistochemical analysis for L-type amino acid transporter 1 (LAT1) was performed in two cases (case 1: Shiba inu, male, aged 14 years, $11 \mathrm{~kg}$; case 2: Shiba inu, female, aged 10 years, $10 \mathrm{~kg}$ ) of surgically resected meningioma. For immunochemical analysis, rabbit anti-canine LAT1 polyclonal antibody was used, donated by Dr Ochiai. This antibody was prepared with the synthetic peptide antigen designed according to the C-terminus amino acid sequence of canine LAT1 (16). Immunohistochemistry was performed as described previously $(17,18)$. Briefly, following deparaffinization, the sections were heated 5 times for $3 \mathrm{~min}$ in a microwave oven in $0.01 \mathrm{M}$ citric acid (pH 6.0) and rinsed with Dulbecco's phosphate-buffered saline (Wako Pure Chemical Industries, Ltd.). Rabbit anti-canine LAT1 polyclonal antibody was incubated with the sections after inactivation of endogenous peroxidase with methanol containing $0.3 \% \mathrm{H}_{2} \mathrm{O}_{2}$ at room temperature for $20 \mathrm{~min}$. Immunostaining was performed using a commercial kit (Histofine Simple Stain MAX PO; Nichirei, Tokyo, Japan). Then, 3,3'-diaminobenzidine (DAB) $\mathrm{H}_{2} \mathrm{O}_{2}$ solution was applied to induce a color development reaction. Following the reaction with DAB, the specimens were washed in deionized water three to four times, followed by staining of the nuclei with hematoxylin for observation.

\section{Results}

Association of PFAA levels with brain tumors in dogs. The results of the PFAA analysis are presented in Fig. 1, where the radar chart presents the percentage of the mean PFAA levels and the PFAA levels of the control group were considered to be $100 \%$. Three amino acids (Ala, Pro and Ile) were significantly increased in the plasma of brain tumor dogs when compared with the control dogs (Ala, $\mathrm{P}=0.03$; Pro, $\mathrm{P}=0.009$; Ile, $\mathrm{P}=0.009$ ). The plasma levels of Ala, Pro and Ile in dogs with brain tumors were increased by 1.6-, 1.5- and 1.6-fold as compared with those in the controls, respectively (Fig. 1). In addition, the brain tumor dogs presented higher Lys and lower Asp levels compared with healthy dogs, although no significant difference was observed. By contrast, the concentrations of the other amino acids (including Arg, Asn, Cys-Cys, Glu, Gln, Gly, His, Leu, Met, Phe, Ser, Thr, Trp, Tyr and Val) remained unchanged between brain tumor and control dogs.

The association of PFAA levels with the type of tumor (glioma or meningioma). As shown in Fig. 2, significantly increased levels of Asn and Gln were observed in the plasma of dogs with glioma when compared with those in dogs with meningiomas $(\mathrm{P}<0.05)$. However, there were no statistically significant changes in other amino acids between dogs with meningioma and those with glioma.

PFAA levels association with idiopathic epilepsy in dogs. As shown in Fig. 1, no significant changes were observed in the levels of any amino acids in dogs with idiopathic epilepsy, as compared with dogs with brain tumors and healthy dogs. Although no significant difference was observed, the levels of 
Table I. Characteristics of dogs included in the present study.

\begin{tabular}{|c|c|c|c|c|}
\hline Breed & Gender & $\begin{array}{c}\text { Age } \\
\text { (years) }\end{array}$ & $\begin{array}{l}\text { Body weight } \\
\qquad(\mathrm{kg})\end{array}$ & Diagnosis \\
\hline French Bulldog & M & 7 & 9 & Brain tumor (glioma) \\
\hline French Bulldog & M & 11 & 10 & Brain tumor (glioma) \\
\hline Shiba Inu & $\mathrm{F}$ & 10 & 7 & Brain tumor (glioma) \\
\hline Golden Retriever & $\mathrm{F}$ & 10 & 26 & Brain tumor (glioma) \\
\hline Mongrel & M & 11 & 27 & Brain tumor (glioma) \\
\hline French Bulldog & M & 9 & 13 & Brain tumor (glioma) \\
\hline Shiba Inu & M & 9 & 15 & Brain tumor (meningioma) \\
\hline Shiba Inu & $\mathrm{F}$ & 10 & 10 & Brain tumor (meningioma) \\
\hline Miniature Schnauzer & $\mathrm{F}$ & 12 & 6 & Brain tumor (meningioma) \\
\hline Shiba Inu & M & 14 & 11 & Brain tumor (meningioma) \\
\hline Yorkshire Terrier & $\mathrm{F}$ & 10 & 2 & Brain metastases of mammary gland tumor \\
\hline Miniature Dachshund & $\mathrm{F}$ & 13 & 4 & Brain metastases of mammary gland tumor \\
\hline Miniature Dachshund & $\mathrm{F}$ & 3 & 3 & Idiopathic epilepsy \\
\hline Beagle & M & 3 & 18 & Idiopathic epilepsy \\
\hline Yorkshire Terrier & M & 6 & 2 & Idiopathic epilepsy \\
\hline Chihuahua & $\mathrm{F}$ & 5 & 3 & Idiopathic epilepsy \\
\hline Toy Poodle & M & 6 & 3 & Idiopathic epilepsy \\
\hline Toy Poodle & $\mathrm{F}$ & 6 & 3 & Idiopathic epilepsy \\
\hline Mongrel & $\mathrm{F}$ & 5 & 3 & Idiopathic epilepsy \\
\hline Mongrel & $\mathrm{F}$ & 10 & 11 & Idiopathic epilepsy \\
\hline Mongrel & M & 14 & 11 & Control \\
\hline Miniature Dachshund & $\mathrm{F}$ & 6 & 4 & Control \\
\hline Toy Poodle & $\mathrm{F}$ & 12 & 3 & Control \\
\hline Chihuahua & $\mathrm{F}$ & 6 & 3 & Control \\
\hline Miniature Dachshund & $\mathrm{F}$ & 7 & 6 & Control \\
\hline Mongrel & M & 12 & 14 & Control \\
\hline Miniature Dachshund & $\mathrm{F}$ & 12 & 6 & Control \\
\hline Pug & $\mathrm{F}$ & 9 & 9 & Control \\
\hline Maltese & $\mathrm{F}$ & 8 & 4 & Control \\
\hline Pekingese & M & 2 & 3 & Control \\
\hline Miniature Schnauzer & $\mathrm{F}$ & 1 & 6 & Control \\
\hline Miniature Dachshund & $\mathrm{F}$ & 14 & 7 & Control \\
\hline Mongrel & M & 1 & 3 & Control \\
\hline Miniature Dachshund & $\mathrm{F}$ & 10 & 8 & Control \\
\hline Chihuahua & M & 10 & 4 & Control \\
\hline Pekingese & M & 6 & 6 & Control \\
\hline
\end{tabular}

F, female; M, male.

Asp obtained from the idiopathic epilepsy dog plasma were lower than those of the healthy dogs.

Immunohistochemistry. Immunohistochemistry of LAT1 localization demonstrated no signal in the tumor cells in case 1 . While in case 2, the tumor cells exhibited a weakly positive reaction.

\section{Discussion}

In the present study, the plasma levels of the amino acids Ala, Pro and Ile in dogs with brain tumor were increased by
1.6-, 1.5- and 1.6-fold, respectively, over the levels reported in healthy dogs (Fig. 1). In addition, the brain tumor dogs presented higher Lys and lower Asp levels compared with healthy dogs, although no significant difference was observed due to variations in the measurements. These results suggest that the PFAA profile in the peripheral blood reflects brain tumor metabolism.

It has been suggested that certain amino acids are associated with human brain tumor metabolism (19). In cerebrospinal fluid (CSF) analysis of human patients with brain tumors, there were significantly higher levels of Pro, Met, taurine, Lys, Ser, 


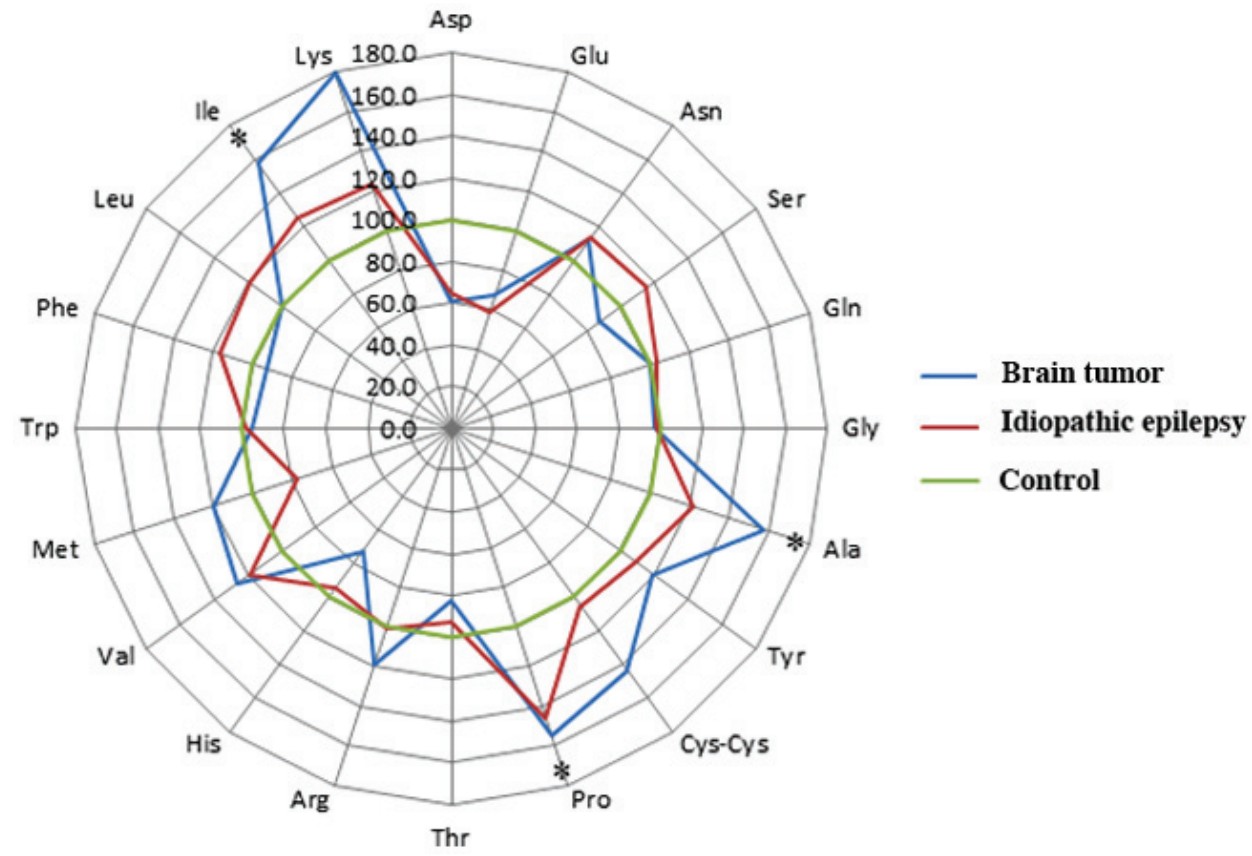

Figure 1. Alterations in PFAA levels in dogs with brain tumors and epilepsy. The radar chart presents the mean PFAA levels, and the PFAA levels of the control group were considered to be $100 \%$. P-values were calculated using the Steel-Dwass test. "P<0.05. PFAA, plasma free amino acid; Ala, alanine; Arg, arginine; Asn, asparagine; Asp, aspartic acid; Cys-cys, cysteine-cysteine; Glu, glutamic acid; Gln, glutamine; Gly, glycine; His, histidine; Ile, isoleucine; Leu, leucine; Lys, lysine; Met, methionine; Phe, phenylalanine; Pro, proline; Ser, serine; Thr, threonine; Trp, tryptophan; Tyr, tyrosine; Val, valine.

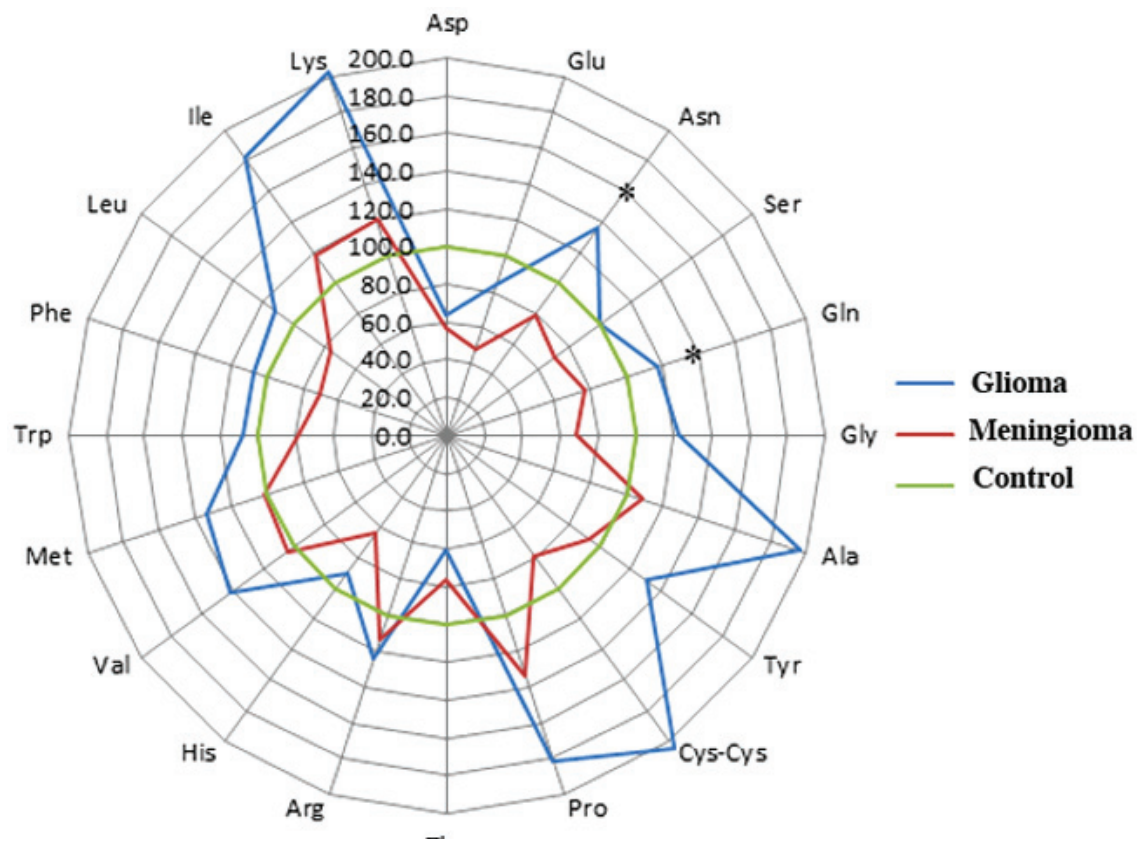

Figure 2. Alterations in PFAA levels in dogs with glioma and meningioma. The radar chart is presents the mean PFAA levels, and the PFAA levels of the control group were considered to be $100 \%$. P-values were calculated using the Steel-Dwass test. "P<0.05. PFAA, plasma free amino acid; Ala, alanine; Arg, arginine; Asn, asparagine; Asp, aspartic acid; Cys-cys, cysteine-cysteine; Glu, glutamic acid; Gln, glutamine; Gly, glycine; His, histidine; Ile, isoleucine; Leu, leucine; Lys, lysine; Met, methionine; Phe, phenylalanine; Pro, proline; Ser, serine; Thr, threonine; Trp, tryptophan; Tyr, tyrosine; Val, valine.

Phe and Gln among patients with malignant glioma compared with those in the controls (19). In another study, amino acid levels in surgically removed glioma and peritumoral tissues were measured, and significantly increased levels of Ile and Val were identified within the peritumoral tissue compared with those in normal brain tissue (13). In MRS studies of human meningioma, increased alanine levels were observed in $91.3 \%$ of cases (20). Although the mechanism underlying these amino acid changes are not clear, they may be due to downregulation of amino acid transporter expression, such as L-type amino acid transporter 1 (LAT1), in tumor cells. In the present study, immunohistochemical analysis for LAT1 was performed in two samples of meningioma, and the results indicated no signal in the samples, which were very weakly stained 
for LAT1 antibody. This suggests that LAT1 may be downregulated in canine brain tumors. Previously, Ochiai et al (16) prepared a synthetic peptide antigen, which was designed according to the $\mathrm{C}$-terminus amino acid sequence of canine LAT1 to investigate LAT1 protein expression in various types of tissue (16). This antibody was used in the present study. Therefore, this antibody is considered to be specific for canine LAT1.

When comparing human patients and dogs with brain tumors, certain amino acids, including Ala, Pro and Ile, were increased in the two species according to the present and previous studies $(13,19,20)$. By contrast, specific amino acids, such as Met, taurine, Lys, Ser, Phe and Gln were increased only in human patients $(13,19)$. This difference between levels in humans and dogs may be due to the source of the sample. In human patients, CSF samples were used (19), whereas plasma obtained from venous blood samples in dogs was used in the present study. It is possible that changes in amino acid levels associated with brain diseases, including brain tumors, would be observed in the CSF as well as in the plasma. To understand the correlation between canine brain tumors and amino acid levels, the associations between plasma and CSF levels of amino acids should be investigated. In addition, the present study demonstrated that Asp levels were decreased in dogs with idiopathic epilepsy, as well as in those with brain tumors, suggesting that the decreased levels of this amino acid may be associated with epileptic seizures, although no evidence is available in the literature.

In veterinary medicine, it has been reported that Thr, Pro and Ser plasma levels were decreased in dogs with melanoma, the Phe and Glu serum levels were increased in dogs with lymphoma, and the Met, Ser, Asn, Gln, Ala, taurine and citrulline plasma levels were decreased in dogs with non-metastatic mammary gland tumors, as compared with these levels in control dogs (8-10). These changes in PFAA levels differed from those observed in the present study, suggesting that the changes noted in the present study were specific to canine brain tumors.

Glu levels in the CSF increased in dogs with idiopathic epilepsy (11). In the present study, PFAA levels were also examined in dogs with idiopathic epilepsy, as well as in healthy dogs and dogs with brain tumors. However, no significant change was observed for any of the amino acids in dogs with idiopathic epilepsy, as compared with the healthy dogs. These findings may be explained based on the functions of the blood-brain barrier (BBB). Amino acid levels may have been altered in the CSF, but not in the peripheral blood of dogs with idiopathic epilepsy, due to the presence of an intact BBB. Generalized convulsive seizures temporarily increase the BBB permeability; however, the loss of BBB integrity recovers within $24 \mathrm{~h}$ (21). In the present study, brain MRI examination was performed in all cases. All dogs with brain tumors showed enhancement in the tumor tissues when using a contrast agent (gadoteridol), suggesting impairment of the BBB. Therefore, the PFAA levels may be altered in dogs with brain tumors (13). Screening of PFAA levels in patients with epileptic seizures may, thus, facilitate the early differential diagnosis of idiopathic epilepsy and symptomatic epilepsy, particularly in the context of brain tumors as a cause of seizures.
An MRS study in humans has suggested that the levels of several metabolites, such as lactic acid and choline, are elevated in malignant gliomas (12). Another MRS study demonstrated no difference in glycine levels between glioblastoma and meningioma patients, although these levels were altered according to the grade of the glioma (22). By contrast, a study of CSF has demonstrated no difference in the amino acid levels in the CSF with the grade of brain tumor (14). In the present study, there were significant differences in the plasma Asn and Gln levels between dogs with meningioma and those with glioma. In addition, in the case of glioma, the levels of Ala, Cys-Cys, Pro, Ile and Lys were higher in brain tumor dogs compared with those in healthy dogs, although there were no statistically significant differences. This result suggests that amino acid levels may be associated with tumor type and grade. Furthermore, it is possible that amino acid levels may change prior to and following treatment, however further investigations are required.

In conclusion, the present study demonstrated that the PFAA levels of dogs with brain tumors were different from those of healthy dogs, with respect to certain amino acids. PFAA levels can be determined by a simple test using blood samples, without the need for special treatments, such as general anesthesia. In addition, multiple types of cancer besides brain tumors can be simultaneously tested for in a single sample, which provides a useful screening test for the early detection of cancer. Future investigations should be conducted in a larger sample size to examine changes and prognoses, depending on tumor types, in order to identify potential diagnostic biomarkers.

\section{Acknowledgements}

The authors would like to thank Dr Ogihara who performed the immunohistochemical analysis.

\section{References}

1. Vandevelde M: Brain tumors in domestic animals. In: Proceedings of a conference on brain tumors in man and animals. Research Triangle Park, NC, 1984.

2. Song RB, Vite $\mathrm{CH}$, Bradley $\mathrm{CW}$ and Cross JR: Postmortem evaluation of 435 cases of intracranial neoplasia in dogs and relationship of neoplasm with breed, age, and body weight. J Vet Intern Med 27: 1143-1152, 2013.

3. Heidner GL, Kornegay JN, Page RL, Dodge RK and Thrall DE: Analysis of survival in a retrospective study of 86 dogs with brain tumors. J Vet Intern Med 5: 219-226, 1991.

4. Snyder JM, Shofer FS, Van Winkle TJ and Massicotte C: Canine intracranial primary neoplasia: 173 cases (1986-2003). J Vet Intern Med 20: 669-675, 2006.

5. LeCouteur RA: Current concepts in the diagnosis and treatment of brain tumours in dogs and cats. J Small Anim Pract 40: 411-416, 1999.

6. Proenza AM, Oliver J, Palou A and Roca P: Breast and lung cancer are associated with a decrease in blood cell amino acid content. J Nutr Biochem 14: 133-138, 2003.

7. Fukutake N, Ueno M, Hiraoka N, Shimada K, Shiraishi K, Saruki N, Ito T, Yamakado M, Ono N, Imaizumi A, et al: A novel multivariate index for pancreatic cancer detection based on the plasma free amino acid profile. PLoS One 10: e0132223, 2015.

8. Azuma K, Osaki T, Tsuka T, Imagawa T, Minami S and Okamoto Y: Plasma free amino acid profiles of canine mammary gland tumors. J Vet Sci 13: 433-436, 2012.

9. Tamai R, Furuya M, Hatoya S, Akiyoshi H, Yamamoto R, Komori Y, Yokoi S, Tani K, Hirano Y, Komori M, et al: Profiling of serum metabolites in canine lymphoma using gas chromatography mass spectrometry. J Vet Med Sci 76: 1513-1518, 2014. 
10. Kawabe M, Baba Y, Tamai R, Yamamoto R, Komori M, Mori T and Takenaka S: Profiling of plasma metabolites in canine oral melanoma using gas chromatography-mass spectrometry. J Vet Med Sci 77: 1025-1028, 2015.

11. Hasegawa T, Sumita M, Horitani Y, Tamai R, Tanaka K, Komori $\mathrm{M}$ and Takenaka S: Gas chromatography-mass spectrometry-based metabolic profiling of cerebrospinal fluid from epileptic dogs. J Vet Med Sci 76: 517-522, 2014.

12. Yamasaki F, Kurisu K, Kajiwara Y, Watanabe Y, Takayasu T, Akiyama Y, Saito T, Hanaya R and Sugiyama K: Magnetic resonance spectroscopic detection of lactate is predictive of a poor prognosis in patients with diffuse intrinsic pontine glioma. Neuro-oncol 13: 791-801,2011.

13. Bianchi L, De Micheli E, Bricolo A, Ballini C, Fattori M, Venturi C, Pedata F, Tipton KF and Della Corte L: Extracellular levels of amino acids and choline in human high grade gliomas: An intraoperative microdialysis study. Neurochem Res 29: 325-334, 2004

14. Piek J, Adelt T, Huse K and Bock WJ: Cerebrospinal fluid and plasma aminograms in patients with primary and secondary tumors of the CNS. Infusionsther Klin Ernahr 14: 73-77, 1987 (In German).

15. Azuma K, Hirao Y, Hayakawa Y, Murahata Y, Osaki T, Tsuka T, Imagawa T, Okamoto Y and Ito N: Application of pre-column labeling liquid chromatography for canine plasma-free amino acid analysis. Metabolites 6: E3, 2016.

16. Ochiai H, Morishita T, Onda K, Sugiyama H and Maruo T: Canine Lat1: Molecular structure, distribution and its expression in cancer samples. J Vet Med Sci 74: 917-922, 2012.
17. Ogihara K, Onda K, Sato R, Naya Y and Ochiai H: Evidence of LAT1 expression in canine caput epididymis. J Vet Med Sci 77: 85-88, 2015.

18. Shi SR, Key MEand Kalra KL: Antigen retrieval in formalin-fixed, paraffin-embedded tissues: An enhancement method for immunohistochemical staining based on microwave oven heating of tissue sections. J Histochem Cytochem 39: 741-748, 1991.

19. Locasale JW, Melman T, Song S, Yang X, Swanson KD, Cantley LC, Wong ET and Asara JM: Metabolomics of human cerebrospinal fluid identifies signatures of malignant glioma. Mol Cell Proteomics 11: 014688, 2012.

20. Demir MK, Iplikcioglu AC, Dincer A, Arslan M and Sav A: Single voxel proton MR spectroscopy findings of typical and atypical intracranial meningiomas. Eur J Radiol 60: 48-55, 2006.

21. Danjo S, Ishihara Y, Watanabe M, Nakamura Y and Itoh K: Pentylentetrazole-induced loss of blood-brain barrier integrity involves excess nitric oxide generation by neuronal nitric oxide synthase. Brain Res 1530: 44-53, 2013.

22. Righi V, Andronesi OC, Mintzopoulos D, Black PM and Tzika AA: High-resolution magic angle spinning magnetic resonance spectroscopy detects glycine as a biomarker in brain tumors. Int J Oncol 36: 301-306, 2010. 